\title{
Implantable Microelectromechanical Sensors for Diagnostic Monitoring and Post-Surgical Prediction of Bone Fracture Healing
}

\author{
Kirk C. McGilvray, ${ }^{1}$ Emre Unal, ${ }^{2}$ Kevin L. Troyer, ${ }^{1}$ Brandon G. Santoni, ${ }^{1}$ Ross H. Palmer, ${ }^{3}$ Jeremiah T. Easley, ${ }^{3}$ \\ Hilmi Volkan Demir, ${ }^{2,4}$ Christian M. Puttlitz ${ }^{1}$ \\ ${ }^{1}$ Orthopaedic Bioengineering Research Laboratory, Departments of Mechanical Engineering and School of Biomedical Engineering, Colorado \\ State University, Fort Collins, Colorado 80523, ${ }^{2}$ Departments of Electrical and Electronics Engineering and Physics, UNAM—Institute of Materials \\ Science and Nanotechnology, Bilkent University, Ankara, Turkey, ${ }^{3}$ Preclinical Surgical Research Laboratory, Department of Clinical Sciences, \\ Colorado State University, Fort Collins, Colorado 80523, ${ }^{4}$ School of Electrical and Electronic Engineering and School of Physical and \\ Mathematical Science, Nanyang Technological University, Singapore
}

Received 12 February 2015; accepted 2 April 2015

Published online 29 July 2015 in Wiley Online Library (wileyonlinelibrary.com). DOI 10.1002/jor.22918

\begin{abstract}
The relationship between modern clinical diagnostic data, such as from radiographs or computed tomography, and the temporal biomechanical integrity of bone fracture healing has not been well-established. A diagnostic tool that could quantitatively describe the biomechanical stability of the fracture site in order to predict the course of healing would represent a paradigm shift in the way fracture healing is evaluated. This paper describes the development and evaluation of a wireless, biocompatible, implantable, microelectromechanical system (bioMEMS) sensor, and its implementation in a large animal (ovine) model, that utilized both normal and delayed healing variants. The in vivo data indicated that the bioMEMS sensor was capable of detecting statistically significant differences $(p$-value $<0.04)$ between the two fracture healing groups as early as 21 days post-fracture. In addition, post-sacrifice microcomputed tomography, and histology data demonstrated that the two model variants represented significantly different fracture healing outcomes, providing explicit supporting evidence that the sensor has the ability to predict differential healing cascades. These data verify that the bioMEMS sensor can be used as a diagnostic tool for detecting the in vivo course of fracture healing in the acute post-treatment period. ( 2015 Orthopaedic Research Society. Published by Wiley Periodicals, Inc. J Orthop Res 33:1439-1446, 2015.
\end{abstract}

Keywords: microelectromechanical system (MEMS); fracture healing; biomechanics; histology; micro computed tomography

It has been shown, in clinical practice and via animal models, that bony healing is critically related to the degree of implant stability and loading. ${ }^{1-3}$ Therefore, the goal of treating displaced or multi-fragmented fractures with orthopedic hardware is to provide adequate biomechanical stability to the fracture site in order to increase the probability that the bone's natural healing process will lead to an acceptable clinical outcome. As normal fracture healing progresses (from an inflammatory phase to granulation tissue formation, cartilage callus formation, and finally, lamellar bone deposition and remodeling) the inherent mechanical stability of the fracture increases. Animal studies, using wired strain gages, have consistently demonstrated that the healing callus and bone assume an increasing proportion of the applied external load as fracture healing proceeds, thus reducing the mechanical burden on the implanted hardware.,5 In abnormal healing (leading to pseudarthrosis or nonunion), this gradual transfer of the loading from the implant to native tissue is significantly altered. In these cases, it is theorized that the healing tissue does not follow the expected reparative cascade, and therefore, cannot assume its temporal load sharing profile

Conflicts of interest: Drs. Demir and Puttlitz are the founding and managing partners of Innovative In Vivo Sensing, LLC, a corporate entity, which holds all intellectual property rights of the bioMEMS technology presented in this manuscript.

Grant sponsor: National Institutes of Health; Grant number: RO1EB010035.

Correspondence to: Christian Puttlitz ( $\mathrm{T}$ : +1-970-491-0956; Fax: +1-970-491-3827; E-mail: puttlitz@engr.colostate.edu)

(C) 2015 Orthopaedic Research Society. Published by Wiley Periodicals, Inc. because it does not have the adequate structural and/ or mineralized tissue component.

Clinicians typically monitor bony healing using diagnostic imaging modalities such as planar radiographic imaging or via physical manipulation. Unfortunately, the course of fracture healing is currently not easily diagnosed in the healing early period, when standard radiographic information regarding the fracture site is not capable of discriminating the healing pathway due to the relative paucity of mineralized tissue. ${ }^{6}$ Manual assessments also suffer from an inherent subjectivity and lack of fidelity. ${ }^{7,8}$ Salvage or revision procedures involving surgery is the most common treatment modality for failed primary operations, and the clinical outcome of these procedures have been negatively correlated to the time between the initial and secondary surgeries due to the temporal course of fibrous tissue accumulation at the fracture site. $^{9}$

Therefore, there is a crucial clinical need to determine the course of bone healing (aberrant versus normal) in the vitally important early stages of fracture management when additional surgery or adjunctive therapies could be implemented with much greater efficacy and result in a much lower risk of clinical failure to the patient. Consequently, new technologies that provide quantitative diagnostic information as to the course of healing during the initial post-operative period could have a significant impact on the treatment of problematic fractures. Accordingly, we developed a biocompatible, microelectromechanical system (bioMEMS) sensor that telemetrically reports data regarding the in vivo mechanical environment of the implant-bone construct, providing a quantitative 
measurement of the relationship between implant mechanics, and ultimately, prediction of the fracture's healing cascade.

\section{MATERIALS AND METHODS BioMEMS Fabrication}

Prior work by our group has undertaken an extensive series of experimental and analytical analyses that included resonance response frequency (RRF) computational models, prototype fabrication, and ex vivo simulations which clearly demonstrated the feasibility of using MEMS technology to telemetrically report on the mechanical environment of the plate-sensor construct by monitoring shifts in the sensor's RRF. ${ }^{10-17}$

There are two separate components to the bioMEMS system: (1) the bioMEMS sensor-implant construct (Fig. 1); and (2) the external excitation/receiving apparatus consisting of an antenna (Fig. 1) attached to a spectrum analyzer. The excitation/receiving antenna emits an electromagnetic wave that induces a current in the bioMEMS sensor. The sensor continuously resonates according to its architectural features. Physical loading of the composite and layered metamaterial sensor results in a change in the sensor's capacitance via physical deformation of the split ring resonator's architecture (Fig. 1). These associated changes in the capacitance $(C)$ of the system are manifested as shifts in the spectral RRF of the sensor (Fig. 1):

$$
\mathrm{RRF}=\frac{1}{2 * \pi * \sqrt{L * C}}
$$

where $L$ represents the sensor's overall inductance. The sensor's architecture was designed such that the RRF output is linearly correlated to the applied physical load and principal strain imparted on the sensor.

The bioMEMS sensors are fabricated using standard MEMS fabrication processes. For biocompatibility, silicon is used as the substrate material of the sensor, gold as the metal layer, and $\mathrm{Si}_{3} \mathrm{~N}_{4}$ as the dielectric layer. ${ }^{10,17}$ The substrate is initially patterned with lithography, and metallization is performed to obtain a film thickness of $0.1 \mu \mathrm{m}$ using $\mathrm{Au}$. The structure is then coated with a $0.1 \mu \mathrm{m}$ thick $\mathrm{Si}_{3} \mathrm{~N}_{4}$ layer using plasma-enhanced chemical vapor deposition (PECVD). Patterning is realized with lithography and exposure is obtained with wet etching. The open portions are metallized with a box coater at a film thickness of $0.1 \mu \mathrm{m}$ $(\mathrm{Au})$. The shape of the device is obtained by a third lithography step, and the process is completed with a $0.1 \mu \mathrm{m}$ thick $\mathrm{Au}$ metallization. The metamaterials architecture is tailored to yield deep and sharp dips at resonance within the transmission frequency spectra (sub-GHz range). The sensor's MEMS architecture has been optimized to generate a high electric field density, which yields amplification of the signal strength and high signal-to-noise ratio (Q-factor ح76). ${ }^{11}$ This process yields a sensor dimension of $8 \mathrm{~mm}$ per side square and $0.8 \mathrm{~mm}$ thick (Fig. 1). For this study, BioMEMS sensors were rigidly adhered to implants (i.e., modified six-hole locking fracture fixation plates; Synthes, West Chester, PA) using a two-part high tensile strength epoxy (2 Ton Clear Epoxy, Devcon, Danvers, MA) (Fig. 1). Prior to implantation, the bioMEMS sensors were coated with medical-grade polyurethane (Master Bond, Inc. Hackensack, NJ).

\section{BioMEMS Sensor Temporal Sensitivity and Biocompatibility Analyses}

In order to simulate the temporal shift in callus tissue stiffness, experiments on an in vitro ovine osteotomy model stabilized with a fracture plate were performed. Temporal alterations in the fracture's stiffness were simulated experimentally by introducing material of increasing stiffness into the osteotomy void. Orthopaedic locking plates (Synthes; $n=6$ per group) were instrumented with a bioMEMS sensor or a standard uniaxial strain gage (V2A-06-062WW-350, Vishay, Raleigh NC). Compression loads (100-900 N) were applied in $100 \mathrm{~N}$ increments using a servo-hydraulic testing system (858 MiniBionix, MTS Systems Corp, Eden Prairie, $\mathrm{MN})$. The testing set-up induced coincident compression and bending. The resultant $\mathrm{RRF}$ shift $(\mathrm{Hz})$ of the bioMEMS
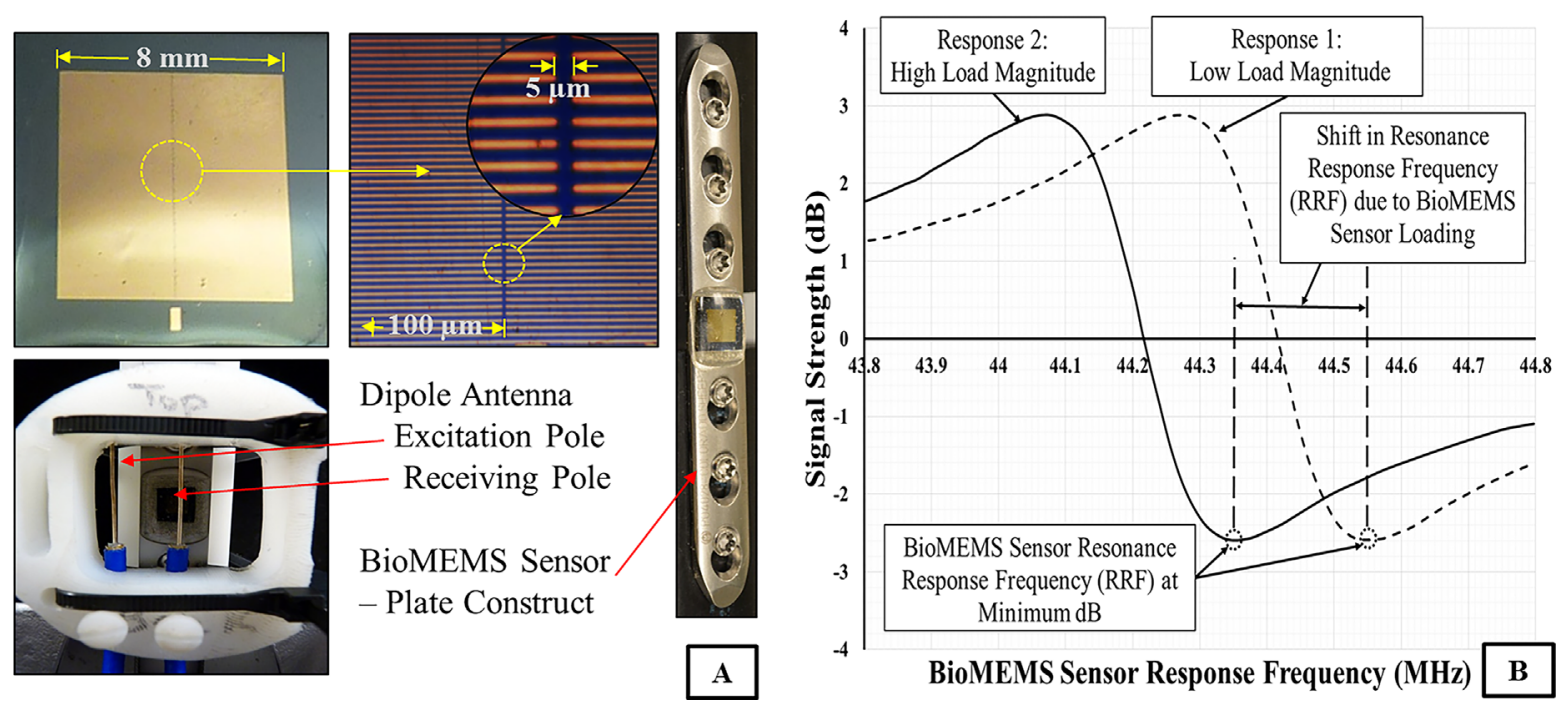

Figure 1. (A) Macro and micro digital images of the bioMEMS sensor, digital image of dipole antenna placed over the bioMEMS sensor; digital image of the BioMEMS sensor-locking plate construct. (B) Characteristic curves of measured signal strength (dB) versus the resonance response frequency (RRF) under applied loading demonstrating the shift in the RRF due to different load magnitudes imparted on the bioMEMS sensor. 
sensor or the micro-strain $(\mu \varepsilon)$ of the uniaxial gage was measured in order to determine the sensor's response as a function of applied strain and osteotomy stiffness.

In order to address the possible confounding issue of temporal degradation of the sensor and/or the epoxy used to bond the sensor to the orthopaedic hardware in a physiological environment, sensor-plate constructs $(n=3)$ were placed in phosphate-buffered saline (PBS) aliquots heated to body temperature $\left(37.0 \pm 2.0^{\circ} \mathrm{C}\right)$ for 90 days. The sensors were removed from their saline environments every two days and the RRF shift was measured under compressive load $(100-900 \mathrm{~N}$ at $100 \mathrm{~N}$ increments; $n=4$ cyclic tests per day per test article). The ratio of the RFF response to the applied load was calculated and compared across the 90 day testing period.

The site-specific biocompatibility and wound-healing response to implantation of the device was also examined. New Zealand white rabbits (Harlan Inc., Indianapolis, IN) $(n=4)$ were implanted with 16 bioMEMS devices and 8 control material implants $\left(\mathrm{Al}_{2} \mathrm{O}_{3}\right.$ as per ASTM standard F603-00) using an intramuscular technique in accordance with ASTM Standards F981-04 and F763-04. Animals were humanely euthanized six months post-operatively in accordance with Colorado State University's Institutional Animal Care and Use Committee (IACUC approval \#07-057A-01). Paraffinembedded slides were created for each implant site and stained with Hematoxylin and Eosin (H\&E). High-resolution digital images were acquired (E800 Microscope, Nikon, Melville, NY) by field for the entire slide section (Spot Imaging Software and Spot Digital Camera, Diagnostic; Media Cybernetic Software, Silver Spring, MD). Slides were analyzed by an experience pathologist to elucidate the severity of the adjacent tissue response to the implanted biomaterials. A standard three-point scale was used to assess tissue response (i.e., inflammatory cells present, fatty infiltration, etc.), with a score of zero indicating no response and a score of three indicating aggressive tissue response.

\section{Signal Attenuation, Antenna Positioning, and Sensor Repeatability Parametric Studies}

A series of parametric studies were performed in order to determine how intervening soft tissues and fiberglass casting material affected the transmission and measurement of the $\mathrm{RRF}$ signal that is emitted from the bioMEMS sensor. Metatarsal osteotomies $(5.1 \pm 0.8 \mathrm{~mm})$ were created in cadaveric ovine hind limbs $(n=4)$ and stabilized using six-hole locking plates (Synthes) that were instrumented with a bioMEMS sensor. Wound closure and fiberglass casting was performed in order to simulate the surgical and post-operative standards of internal fracture reduction care. Compression loads (100-900 N) were applied in $100 \mathrm{~N}$ increments to the limb using a servo-hydraulic testing system (858 MiniBionix, MTS Systems Corp) and the RRF shift of the sensor was measured. Using an adjustable $x-y$ table, the spatial relationship between the poles and the sensor was manipulated in three dimensions. Ovine cadaveric tissue (vastus lateralis muscle, epidermis, and dermis) and fiberglass casting material thicknesses were increased between the sensor and the excitation/receiving antenna until the measured RRF signal strength (dB) fell below an acceptable level $(<0.001 \mathrm{~dB})$. Parametric studies investigating the functional relationships with respect to the spatial coordinates between the antenna's excitation/receiving poles and the intervening tissue and the sensor's response were performed.

\section{In Vivo Evaluation}

Following rigorous in vitro analyses and a comprehensive biocompatibility study, an in vivo large animal (ovine) study was initiated. We hypothesized that the healing cascade in the critically important early period (7-35 days post-surgery) could be determined by temporally monitoring the bioMEMS sensor's RRF, and that these quantitative changes in the sensor's response would be predictive of normal and aberrant bone fracture healing.

Fourteen skeletally mature sheep (Columbia x Rambuillet cross breed, $\sim 3.5$ years old) were utilized in this study (IACUC approval \# 09-1471A). The surgery site was aseptically prepared using standard practices. The metatarsal was exposed through an insertion in the lateral part of the left hind limb. The bioMEMS sensor-locking plate construct (Synthes) was placed on the metatarsal and the required holes were drilled under saline irrigation. Self-tapping locking screws (DLS System, Synthes) were used attach the hardware to the metatarsal. An oscillating bone saw was used to create the planned ostectomy with saline irrigation to reduce the possibility of thermal necrosis. Two osteotomy models were utilized (union and non-union). Pilot work $(n=2)$ by our group showed that an in vivo ovine metatarsal osteotomy larger than $10.27 \pm 1.25 \mathrm{~mm}$ would not form a mechanically stable callus after 6 weeks of healing, and thus this fracture gap dimension was used as a lower bound to create a non-union sized defect. Defects were classified as an expected union ( $n=7 ; 4.8 \pm 1.2 \mathrm{~mm}$ osteotomy) when calcified tissue was expected to bridge the osteotomy after approximately 6 weeks of healing. Non-union sized defects $(n=7$; $13.8 \pm 1.0 \mathrm{~mm}$ osteotomy) were classified as constructs wherein it was expected that calcified tissue would not bridge the osteotomy after 6 weeks of healing. These ovine osteotomy fracture models were deemed analogous to union and nonunion human long bone fracture conditions. The sheep were free to ambulate ad arbitrium after the surgery in order to allow post-surgical weight-bearing of the fracture site. Biweekly RRF data were recorded from the implanted sensor while the treated limb was serially loaded in compression $(50-450 \mathrm{~N})$ using a custom-designed fixture. Six loading cycles were performed per testing day; daily RRF means and standard deviations per treatment group were calculated.

Following euthanasia, the metatarsi were carefully collected and the bioMEMS plate constructs were removed from the underlying bone. Metatarsals were then placed in $10 \%$ phosphate buffered formalin. Micro-computed tomography $(\mu \mathrm{CT})$ analyses were performed at the mid-diaphyseal fracture (voltage: $70 \mathrm{kVp}$, current: $114 \mu \mathrm{A}$, integration time: $500 \mathrm{~ms}$; Scanco $\mu \mathrm{CT}$ 80, Sanco Medical AG, Bruttisellen, Switzerland). $\mu \mathrm{CT}$ data were rendered into three-dimensional images and mid-sagittal slices were examined for continuous bone bridging between osteotomy cuts.

Following $\mu \mathrm{CT}$ analyses, the treated metatarsi were processed for decalcified histological analysis. Samples were placed in decalcifying solution following the initial fixing period until fully decalcified as verified via radiographic examination. Samples were subsequently dehydrated in graded solutions of ethanol (ETOH), infiltrated with paraffin on a tissue processor (Tissue-Tek VIP, Sakura, Torrance, CA), and embedded using standard paraffin histology techniques (Histocentre 2, Thermo Shandon Inc, Pittsburgh, PA). Histological sections were taken in the transverse (mediolateral) plane to include the fracture site and associated callus of the diaphyseal region of the metatarsus. The decalcified paraffin blocks were faced and 
two 8 micron sections were cut from each limb on a rotary microtome (RM2255 Leica, Nussloch, Germany) and stained with Hematoxylin and Eosin. High-resolution digital images were acquired for histomorphometric analysis using a Spot Imaging System (Diagnostic Instruments, Sterling Heights, MI), a microscope (E800, Nikon, AG Heinze, Lake Forest, CA), and analyzed with commercially available histomorphometry software (Image Pro, Media Cybernetics, Silver Spring, MD). Histomorphometric images (at $10 \times$ magnification) were acquired and area fractions of mineralized and fibrous tissue were calculated in the periosteal callus, diaphyseal cortices, and intramedullary canal. Total periosteal callus bone and fibrous tissue areas $\left(\mathrm{mm}^{2}\right)$ were determined by selecting and segmenting callus material contralateral to the orthopaedic plate on the periosteal surface of the bone within the original osteotomy boundaries. Bone and fibrous tissue area percentages (\%) were calculated within the periosteal callus, diaphyseal cortex, and intramedullary canal by normalizing the ratio of segmented tissue area to the total ROI.

\section{Statistical Analyses}

Since all data passed normality and equal variance testing, statistical differences were detected between the treatment groups using a standard Student's $t$-test (SigmaPlot, San Jose, CA), where a $p$-value less than 0.05 was considered statistically significant. Statistical correlations were quantified using Pearson product moments (SigmaPlot).

\section{RESULTS \\ BioMEMS Sensor Temporal Sensitivity and Biocompatibility Analyses}

In vitro experiments utilizing an ex vivo ovine metatarsal fracture model that had been surgically stabilized indicated that, by monitoring the RRF of the bioMEMS sensor-plate constructs, it was possible to correlate hardware strain $(\mu \varepsilon)$ to discrete changes in the systems RRF response $(\mathrm{Hz})$ under complex loading conditions (bending and compression). The bioMEMS sensor demonstrated a highly linear relationship to implant principal strain (Pearson's correlation coefficient $=0.95 \pm 0.05$ ), with a sensing resolution (i.e., RRF shift) of $33.73 \pm 2.44 \mathrm{~Hz} / \mu \varepsilon$. Examination of the sensitivity between the sensor's RRF response $(\mathrm{Hz})$ and the applied load $(\mathrm{N})$ indicated that the bioMEMS sensor could detect stiffness changes of greater than $0.5 \mathrm{~N} / \mathrm{mm}$ within the simulated fracture gap.

Repeatability experiments indicated that there was no significant change in the sensor's RRF under mixed loading following prolonged exposure to heated $\left(37.0 \pm 2.0^{\circ} \mathrm{C}\right)$ physiologic saline environment over a 90 day period (Fig. 2). The data demonstrated a mean ratio of $\mathrm{RRF}$ to applied load of $34.35 \pm 3.16 \mathrm{~Hz} / \mathrm{N}$ over the duration of the experiment. No statically significant change in the sensor's RRF response was observed $(p=0.80)$, with an average response of $34.17 \pm 2.64 \mathrm{~Hz} / \mathrm{N}$ on day 1 and $34.83 \pm 3.43 \mathrm{~Hz} / \mathrm{N}$ on day 90 (Fig. 2).

Biocompatibility experiments utilizing a rabbit model confirmed that there was no adverse tissue reaction to the bioMEMS materials either immediately adjacent to or at locations remote from the implantation site.
Upon retrieval, there was no gross evidence of corrosion/oxidation on the bioMEMS sensor surfaces. Semi-quantitative histopathologic examination of tissue-sensor sections confirmed the absence of abnormal macrophage or lymphocytic cellular activity. Minimal tissue fibrosis was measured surrounding both the control $\left(\mathrm{Al}_{2} \mathrm{O}_{3}\right.$ coupons $)$ and bioMEMS sensors $(1.0 \pm 0.5$ and $0.9 \pm 0.2$, respectively, using a standard 3 -point scale), and the general toxicity score for the test and control materials were both zero.

\section{Signal Attenuation, Antenna Positioning, and Sensor Repeatability Parametric Studies}

The RRF data demonstrated the linearity of the sensor's response to mixed mechanical loading (Pearson's correlation coefficient $=0.91 \pm 0.03$ ) when the signal was transmitted though biological soft tissue. In addition, the sensor's sensitivity was highly repeatable, with the resonant frequency varying less than $5 \%$ with respect to the mean $R R F$ value $(32.27 \pm 1.60 \mathrm{~Hz} / \mathrm{N})$. These experiments demonstrated that the measured signal strength was maintained until the soft tissue thickness was increased to $30.00 \pm 1.30 \mathrm{~cm}$. These constructs also included $1.65 \pm 0.25 \mathrm{~cm}$ of casting material on the periphery of the soft tissue.

The spatial orientation between the excitation and receiving poles of the antenna's dipole configuration demonstrated an optimal spatial separation of $15.00 \pm 1.75 \mathrm{~mm}$. It was determined that a straight and parallel dipole antenna configuration produced the best transmission and reception characteristics, with low background noise $(0.18 \pm 0.12 \mathrm{~dB})$ and reception $(2.64 \pm 1.12 \mathrm{~dB})$ signal strengths.

\section{In Vivo Evaluation}

The in vivo data indicated a reduction in the measured $\mathrm{RRF}$ with respect to the applied load $(\mathrm{RRF} / \mathrm{N})$ as healing time increased ([RRF/N]/day) (Fig. 3). These gradual decreases in the $\mathrm{RRF}$ over time reflect reductions in hardware strain due to fracture stabilization and healing, and closely mirror previously reported wired strain gage studies. ${ }^{4,5,18}$ The $\mathrm{RRF} / \mathrm{N}$ data (calculated between days 7 and 30 post-surgery) was used to compare the sensor's differential response between the two treatment groups during the acute healing period (Fig. 3). The in vivo data indicated that the bioMEMS sensor was capable of detecting statistically significant differences ( $p$-value $<0.04$ ) between the two fracture healing groups as early as 21 days post-fracture. These RRF data also indicated a statistically significant difference $(p=0.01)$ between the union $(-10.69 \pm 1.79$ $[\mathrm{Hz} / \mathrm{N}] /$ day $)$ and non-union $(-4.87 \pm 0.59[\mathrm{~Hz} / \mathrm{N}] /$ day $)$ models during the initial 30 day healing period (Fig. 3). This $220 \%$ difference in the rate of change in hardware strain within the acute fracture healing period for the union sized osteotomy group indicated the fracture callus in this group had a greater capacity to support limb loading and stabilize the fracture. 
Temporal Shift in RRF Response in Heated Saline

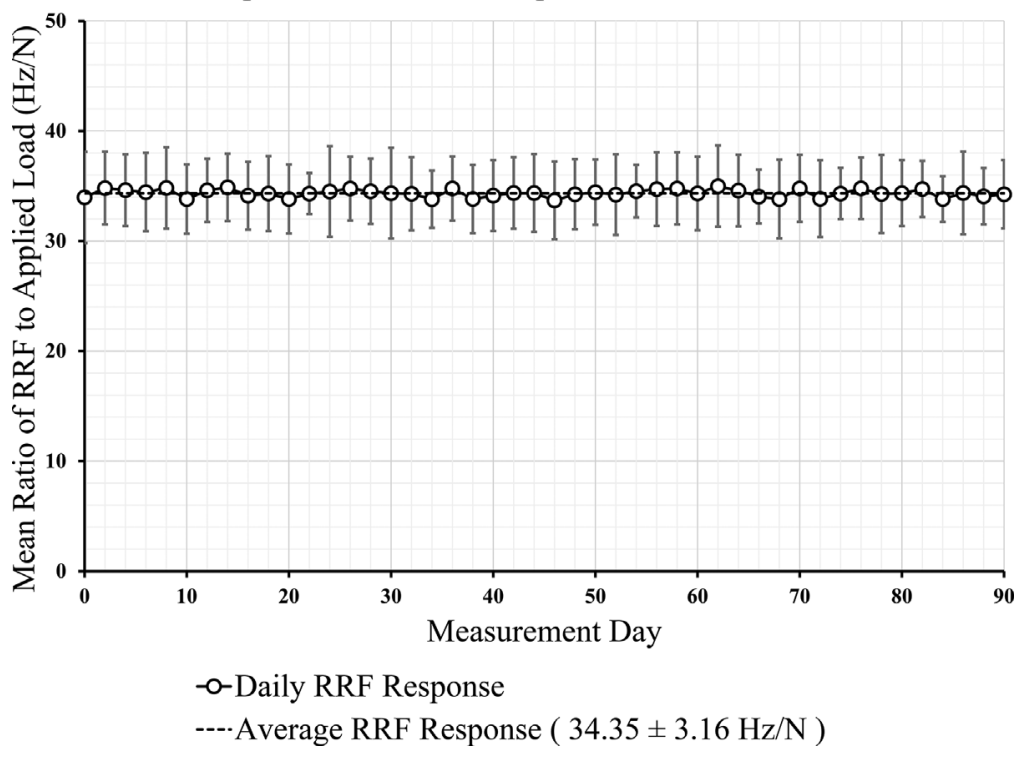

Figure 2. Repeatability experiments indicated that there was no significant change or shift in the sensor's RRF response under mixed loading following prolonged exposure to heated $\left(37.0 \pm 2.0^{\circ} \mathrm{C}\right)$ physiologic saline environment over a 90 day period.
Thresholding and segmentation analyses of weekly radiographic images of the fracture confirmed the differential healing response between the two groups (Fig. 4). The non-union osteotomy group had little or no visible calcified tissue accumulation across the facture site; the percentage of calcified tissue within the region of interest (i.e., osteotomy plus callus area) ranged from $2.1 \pm 4.0 \%$ at 7 days post-surgery to $10.4 \pm 20.1 \%$ at the time of sacrifice (day $45 \pm 8$ ). Conversely, the union osteotomy group demonstrated complete calcified tissue bridging of the osteotomy, with the calcified tissue component representing $2.8 \pm 4.5 \%$ at 7 days post-surgery and $44.7 \pm 27.7 \%$ at the time of sacrifice (day $45 \pm 8$ ). Correlation between the radiographic analyses and the $R R F$ response was not observed during the acute healing phase for either group ( $p$-value $=0.99$ ), providing further confirmation that standard radiography cannot predict the course of fracture healing in the acute healing phase.

Post sacrifice ( $45 \pm 8$ days post-surgery) $\mu \mathrm{CT}$ reconstructions visually demonstrated complete continuous calcified tissue bridging the entire osteotomy site for all $(n=7 / 7)$ of the union samples and none $(n=0 / 7)$ of the non-union surgical fractures (Fig. 4). Bone histopathology measurements within the area encompassing the osteotomy and the callus region (i.e., the region of interest, ROI) corroborated these $\mu \mathrm{CT}$ data (Fig. 4). The non-union group had statistically less $(p<0.01)$ bone $(11.4 \pm 23.3 \%$ area of bone within the ROI) at the fracture site, a $73 \%$ decrease in mineralized tissue as compared to the union model $(53.0 \pm 28.5 \%$ area of bone within the ROI) group. A statistically significant
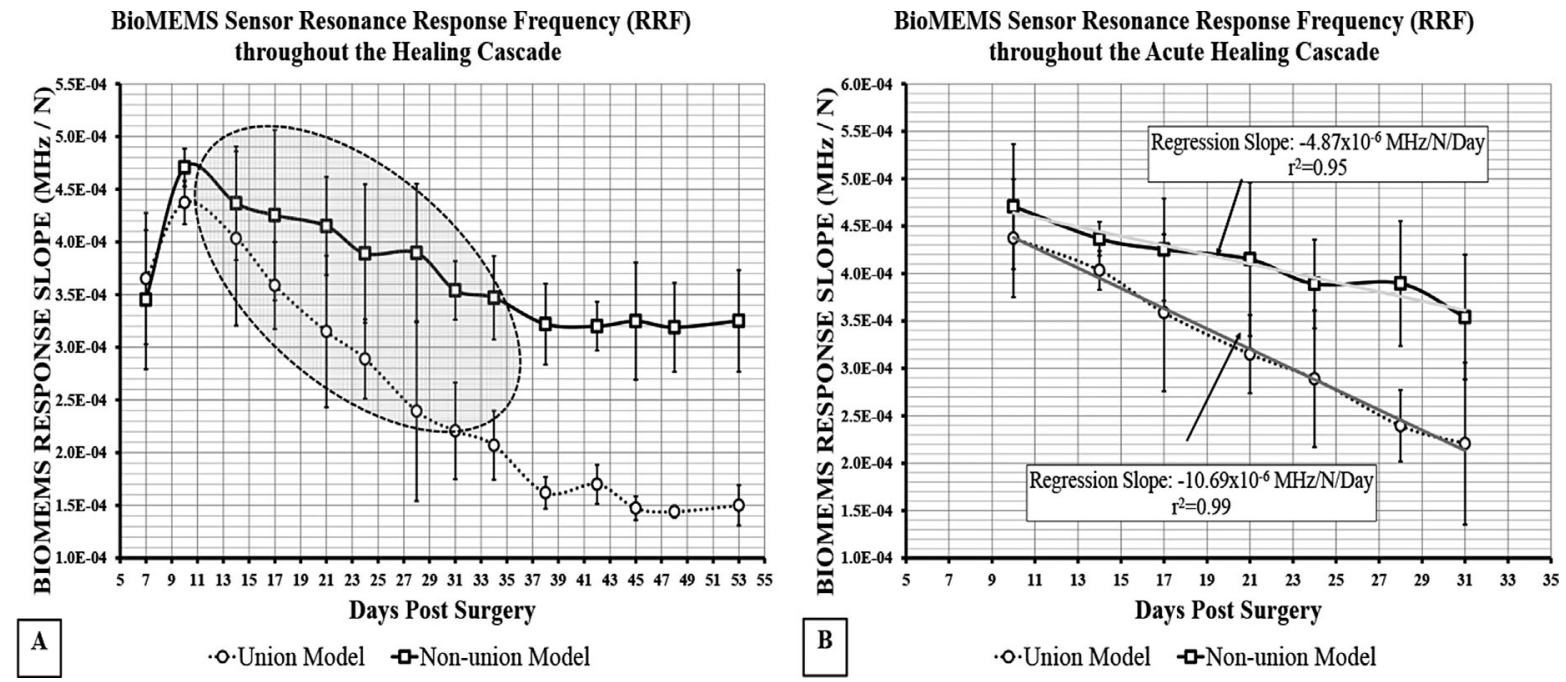

Figure 3. (A) BioMEMS sensor's mean (w/one standard deviation error bars) temporal RRF response observed under loading during the healing cascade in an ovine metatarsal model. (B) The response during the acute healing phase (7-30 days post-surgery) demonstrated a statistically significant difference $(p=0.01)$ between the union $(-10.69 \pm 1.79[\mathrm{~Hz} / \mathrm{N}] /$ Day $)$ and non-union $(-4.87 \pm 0.59$ $[\mathrm{Hz} / \mathrm{N}] /$ Day) models, indicating a substantial temporal reduction in hardware strain in the union model. 
Union Model

A

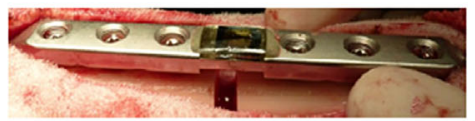

$\mathrm{B}_{1}$

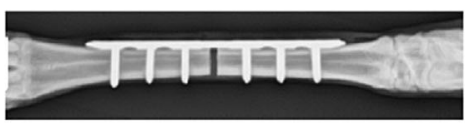

$\mathrm{B}_{2}$

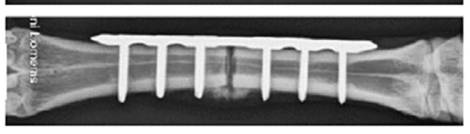

$\mathbf{B}_{3}$
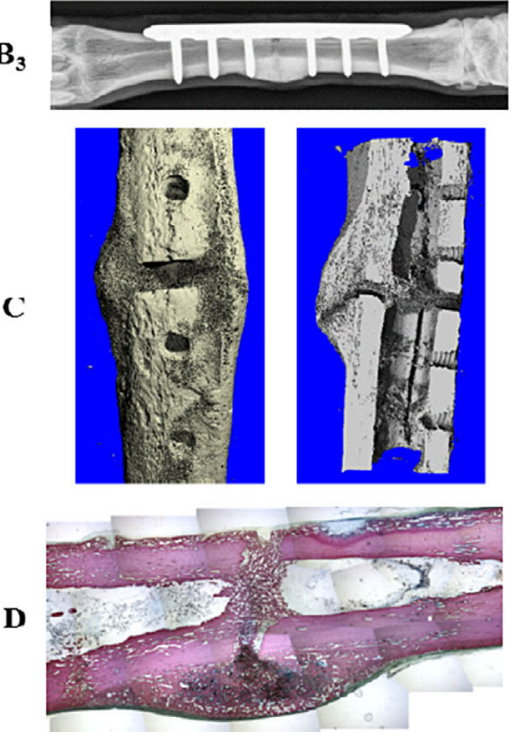

\section{Non-union Model}
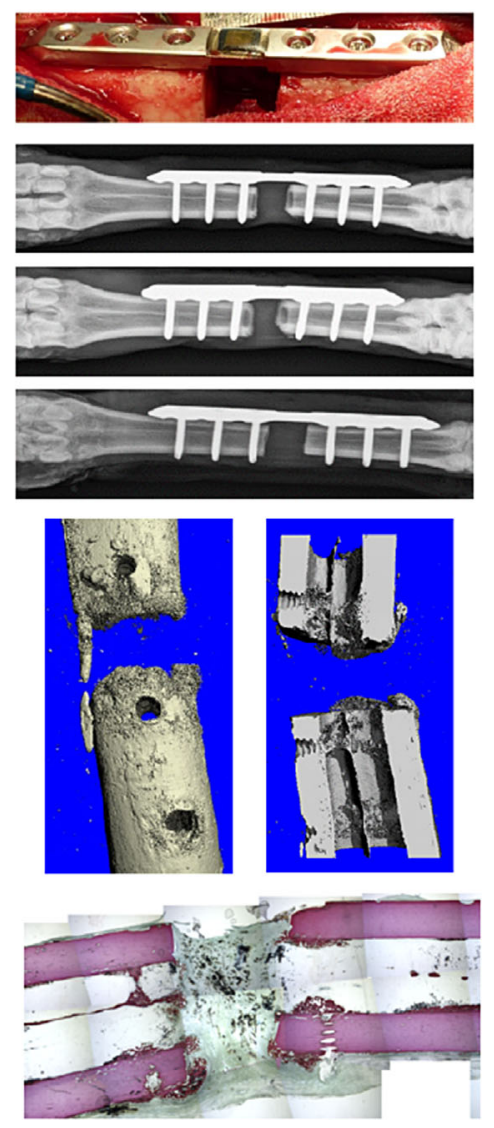

Figure 4. (A) Digital images obtained at the time of surgery that demonstrate the different osteotomy gaps used to model the union $(4.8 \pm 1.2 \mathrm{~mm})$ and nonunion $(13.8 \pm 1.0 \mathrm{~mm}$ ) healing conditions. (B) Radiographs demonstrating the different model variants at $\left(\mathrm{B}_{1}\right) 7$ days, $\left(\mathrm{B}_{2}\right) 24$ day, and $\left(\mathrm{B}_{3}\right) 45$ days. Calcified tissue within the non-union osteotomy ranged from $2.1 \pm 4.0 \%$ at 7 days post-surgery to $10.4 \pm 20.1 \%$ at the time of sacrifice. Calcified tissue within the union osteotomy ranged from $2.8 \pm 4.5 \%$ at 7 days postsurgery and $44.7 \pm 27.7 \%$ at the time of sacrifice (C) $\mu \mathrm{CT}$ reconstructions demonstrated complete continuous calcified tissue bridging the entire osteotomy site for all $(n=7 / 7)$ of the union samples and none $(n=0 /$ 7) of the non-union surgical fractures (day 45 postsurgery). (D) Histology sections stained with H\&E to highlight the fibrous (gray stain) and calcified (red stain) tissue constituents. Pathology report concluded that all histologic changes were mild and consistent with incidental findings. correlation between the bioMEMS sensor response and percent bone at the facture site for both the union (Pearson's correlation coefficient: $-0.97 ; \quad p$-value $<0.01$ ) and non-union groups (Pearson's correlation coefficient: $-0.96 ; p$-value $<0.01)$ was determined. These correlations indicated that the sensor has the ability to inform on the amount of bone present within the fracture gap, which is indicative of the relative biomechanical stability associated with the healing callus.

Paraffin-embedded biocompatibility histological analyses of the peri-surgical, soft tissues, and remote organs were also performed. The pathology report concluded that all histologic changes were mild and consistent with incidental findings.

\section{DISCUSSION}

The clinically available options to assess acute osteosynthesis stability due to fracture healing are limited. The course of healing is usually estimated by evaluation of temporal radiographs and is subjective in nature, making it difficult to quantitatively assess the extent of fracture stability. ${ }^{19}$ Clinical experience, as well as scientific investigation, has shown substantial inter-observer variability in judging healing using currently available imaging modalities, ${ }^{20,21}$ and it has been shown that it is exceedingly difficult to correlate the radiographic appearance of the fracture site with mechanical stability. ${ }^{22,23}$ Additionally, during the relatively slow process of primary bone healing, there are little to no visible changes on plain radiographs. ${ }^{24}$ Thus, there is a critical need to discern the mechanical stability of the fracture site prior to callus calcification. ${ }^{25}$ As healing progresses it is advantageous to quantify implant load, because not only does the plate load reflect the size of the callus, but also the degree of mineralization within the fracture site. ${ }^{5}$ Our measurements showed that stability follows a continuous function, with biomechanical changes beginning well before they can be detected by radiographic imaging. If clinically available, these data could allow practitioners to optimize postoperative treatment regimens and avoid pseudarthrosis. Specifically, sequential bioMEMS RRF data could be used to indicate when a wide spectrum of minimally invasive (i.e., nonsurgical) adjunctive therapies, including injections of osseous "biological" therapeutics, bone morphogenetic proteins $(\mathrm{BMPs})^{26,27}$ or other growth factors that potentiate the osteoinductive activities of BMPs, ${ }^{28-31}$ should be administered.

Similar patterns of decreasing implant strain magnitude during the healing cascade have been observed via telemetric assessments of internal femoral fixator in humans, ${ }^{21}$ telemetric assessments of 
internal intramedullary nails in humans,${ }^{32,33}$ wired external fixators in sheep ${ }^{4}$ telemetric assessment in femoral replacements in humans, ${ }^{34,35}$ fixation plates with a wired strain gages in sheep, ${ }^{5}$ and wired external fixators in humans. ${ }^{18,36}$ These studies support the contention that significant changes in osteosynthesis stiffness during the acute healing phase (days 1-30) transpire well before radiographic observations (i.e., plain radiographs and coronal CT images) of calcified tissue can be obtained. Our data further support these findings wherein the captured sequential radiographic imaging demonstrated no correlation in the acute healing phase for either treatment group. Conversely, the RRF bioMEMS sensor data quantitatively described mechanical load sharing changes between the implant and the native tissue during the critically-important, acute, postoperative period of the healing cascade by demonstrating statistically significant differences in the sensor's response between the union and non-union groups.

Although a variety of methods are available to directly or indirectly determine the biomechanical characteristics of a healing fracture, most of these methods come with considerable limitations, ${ }^{25}$ which yields their routine implementation in the clinical milieu challenging. However, the specific MEMS structure, metamaterial architecture, and radio frequency technology used in the current study are advantageous for in vivo medical applications because: (1) the sensor can be inductively powered obviating the need for an implantable power source; (2) the sensor can sense and transmit the in vivo biological data wirelessly, eliminating the internal-external physical connection; and (3) the sensor's size can be miniaturized allowing for its use in applications that require small dimension. We anticipate that with further experience with this device, it will be possible to predict complete fracture healing or eventual non-union based on the temporal mechanics of the fracture site.

Implantable MEMS devices for in vivo monitoring are a rapidly evolving field, with a constant stream of technologies being developed. MEMS devices including conventional pacemakers, implantable defibrillators, heart pressure monitors, and drug delivery devices have shown a great potential in the application of individualized medicine. Therefore, we envision that the developed device described herein has the potential to be translated for clinical applications.

\section{AUTHOR'S CONTRIBUTIONS}

All authors have read and approved the final submitted manuscript. All authors contributed equally.

\section{REFERENCES}

1. Dickson K, Katzman S, Delgado E, et al. 1994. Delayed unions and nonunions of open tibial fractures. Correlation with arteriography results. Clin Orthop Relat Res 189-193.
2. Miclau T, Lu C, Thompson Z, et al. 2007. Effects of delayed stabilization on fracture healing. J Orthop Res 25:1552-1558.

3. Thompson Z, Miclau T, Hu D, et al. 2002. A model for intramembranous ossification during fracture healing. J Orthop Res 20:1091-1098.

4. Grasa J, Gomez-Benito MJ, Gonzalez-Torres LA, et al. 2010. Monitoring in vivo load transmission through an external fixator. Ann Biomed Eng 38:605-612.

5. Stoffel K, Klaue K, Perren SM. 2000. Functional load of plates in fracture fixation in vivo and its correlate in bone healing. Injury 31:B37-B50.

6. Schmidhammer R, Zandieh S, Hopf R, et al. 2004. Alleviated tension at the repair site enhances functional regeneration: the effect of full range of motion mobilization on the regeneration of peripheral nerves-histologic, electrophysiologic, and functional results in a rat model. J Trauma 56:571-584.

7. Schmickal T, von Recum J, Wentzensen A. 2005. Stiffness measurement of the neocallus with the Fraktometer FM 100. Arch Orthop Trauma Surg 125:653-659.

8. Webb J, erling G, Gardner T, et al. 1996. Manual assessment of fracture stiffness. Injury 27:319-320.

9. Garcia P, Holstein JH, Maier S, et al. 2008. Development of a reliable non-union model in mice. J Surg Res 147:84-91.

10. Melik R, Perkgoz NK, Unal E, et al. 2008. Bio-implantable passive on-chip RF-MEMS strain sensing resonators for orthopaedic applications. J Micromech Microeng 8:1-9.

11. Melik R, Unal E, Perkgoz NK, et al. 2009. Circular high-Q resonating isotropic strain sensors with large shift of resonance frequency under stress. Sensors 9:9444-9451.

12. Melik R, Unal E, Perkgoz NK, et al. 2009. Flexible metamaterials for wireless strain sensing. Appl Phys Lett 95:5000-5007.

13. Melik R, Unal E, Perkgoz NK, et al. 2009. Metamaterialbased wireless strain sensors. Appl Phys Lett 95:19609-19621.

14. Melik R, Unal E, Perkgoz NK, et al. 2010. Metamaterial based telemetric strain sensing in different materials. Optics Express 18:5000-5007.

15. Melik R, Unal E, Perkgoz NK, et al. 2010. Metamaterialbased wireless RF-MEMS strain sensors. Ieee Sensors 2010:2173-2176.

16. Melik R, Unal E, Perkgoz NK, et al. 2011. RF-MEMS load sensors with enhanced Q-factor and sensitivity in a suspended architecture. Microelectron Eng 88:247-253.

17. Melik R, Unal E, Perkgoz NK, et al. 2010. Nested metamaterials for wireless strain sensing. IEEE J Sel Top Quantum Electron 16:450-458.

18. Claes LE, Cunningham JL. 2009. Monitoring the mechanical properties of healing bone. Clin Orthop Relat Res 467: 1964-1971.

19. Morshed S, Corrales L, Genant H, et al. 2008. Outcome assessment in clinical trials of fracture-healing. J Bone Joint Surg Am 90:62-67.

20. Bourgois R, Burny F. 1972. Measurement of the stiffness of fracture callus in vivo. A theoretical study. $J$ Biomech $5: 85-91$.

21. Seide K, Aljudaibi M, Weinrich N, et al. 2012. Telemetric assessment of bone healing with an instrumented internal fixator: a preliminary study. J Bone Joint Surg Br 94: 398-404.

22. Davis BJ, Roberts PJ, Moorcroft CI, et al. 2004. Reliability of radiographs in defining union of internally fixed fractures. Injury 35:557-561.

23. Ogrodnik PJ, Moorcroft CI, Thomas PB. 2007. Measuring multi-dimensional, time-dependent mechanical properties of a human tibial fracture using an automated system. Proc Inst Mech Eng H 221:641-652. 
24. Hak DJ, Fitzpatrick D, Bishop JA, et al. 2014. Delayed union and nonunions: epidemiology, clinical issues, and financial aspects. Injury 45:S3-S7.

25. Augat P, Faschingbauer M, Seide K, et al. 2014. Biomechanical methods for the assessment of fracture repair. Injury 45 : S32-S38.

26. Celeste AJ, Iannazzi JA, Taylor RC, et al. 1990. Identification of transforming growth factor beta family members present in bone-inductive protein purified from bovine bone. Proc Natl Acad Sci USA 87:9843-9847.

27. Wozney JM, Rosen V, Celeste AJ, et al. 1988. Novel regulators of bone formation: molecular clones and activities. Science 242:1528-1534.

28. Joyce ME, Jingushi S, Bolander ME. 1990. Transforming growth factor-beta in the regulation of fracture repair. Orthop Clin North Am 21:199-209.

29. Nakamura T, Hara Y, Tagawa M, et al. 1998. Recombinant human basic fibroblast growth factor accelerates fracture healing by enhancing callus remodeling in experimental dog tibial fracture. J Bone Miner Res 13:942-949.
30. Nash TJ, Howlett CR, Martin C, et al. 1994. Effect of platelet-derived growth factor on tibial osteotomies in rabbits. Bone 15:203-208.

31. Trippel SB. 1998. Potential role of insulinlike growth factors in fracture healing. Clin Orthop Relat Res 355:S301-S313.

32. Wilson DJ, Morgan RL, Hesselden KL, et al. 2009. A singlechannel telemetric intramedullary nail for in vivo measurement of fracture healing. J Orthop Trauma 23:702-709.

33. Schneider E, Michel MC, Genge M, et al. 2001. Loads acting in an intramedullary nail during fracture healing in the human femur. J Biomech 34:849-857.

34. Lu TW, O'Connor JJ, Taylor SJ, et al. 1998. Validation of a lower limb model with in vivo femoral forces telemetered from two subjects. J Biomech 31:63-69.

35. Shah AD, Taylor SJ, Hua J. 2006. Correlation of radiographic and telemetric data from massive implant fixations. J Biomech 39:1304-1314.

36. Al Nazer R, Lanovaz J, Kawalilak C, et al. 2012. Direct in vivo strain measurements in human bone-a systematic literature review. J Biomech 45:27-40. 